\title{
Mechanistic and topological explanations: an introduction
}

In the last twenty years, especially after the publication of a seminal paper by Watts and Storgatz (1998), an interest in network methods has spread like a wild fire over many areas of science, e.g. ecology, evolutionary biology, medicine, and cognitive neuroscience (Bassett and Bullmore 2006; Bullmore and Sporns 2009; Sporns 2012; Benkö et al, 2009; 2009; Cupal et al, 1999; Fontana 2002; Barabasi and Albert, 1999; Barabasi and Oltvai, 2004; Barabasi et al, 2011). Those methods have been seen by some philosophers as supporting a distinct kind of explanation that they call topological explanation (Huneman 2010).

The areas of science in which topological explanations are used, are traditionally dominated by other kinds of explanations, e.g. mechanistic, dynamical, probabilistic, functional, causal, semantic. The rising importance of network modelling in social science, cell biology, molecular genomics (think of Gene Regulatory Networks) or neuroscience, where methodology is largely based on network analysis and the graph-theoretical properties are treated as a kind of topological properties, as well as the pervasive use of landscape models for states of systems (inherited from physics, and now pervasive in population genetics, ecology or in the study of cell metabolism), are tokens of the fruitfulness of topological explanatory practices. This philosophically unexplored area provides a new perspective on traditional issues in philosophy of science, such as explanation, realization relation, validation, levels of explanation, and many others.

Very abstractly, the mechanistic explanation describe entities and activities that are organized to produce something or to perform a process or a function. Different levels of organization or properties of the same system can be explained by describing different mechanisms. Mechanistic philosophers of science have made an important point in several landmark publications (Bechtel and Richardson 1993; Machamer, Darden, Craver 2000; Craver 2007, 2013) by showing that in biological sciences and neuroscience, classical models of explanation such as the Hempelian D-N model, that were thought to reflect the explanatory practices mostly taken from physics, are not adequate for explaining biological phenomena and their complexity.

In contrast, topological explanations, explain by a reference to structural or mathematical properties of the system (e.g. graph-theoretical properties, topological features, or properties of mathematical structures in general), and abstract away from the details of particular causal interactions or mechanisms. There are varieties of such explanations, that have been termed in various ways depending on the nature of the features relevant to the explanation: "program explanations" (Jackson \& Pettit 1990), "topological explanations" (Huneman 2010); "mathematical explanations" (Batterman 2010). This special issue treats specifically explanations that are based on network and graph-theoretical methodology in sciences (broadly construed), and which are also referred to in the scientific literature as the topological explanations.

Neither the relationship between these forms of explanations, nor their relative prospects, have yet been widely discussed. This is particularly surprising given that, during the last twenty years topological approach has gained its popularity in evolutionary biology and ecology and in the last ten years in cognitive neuroscience, and in particular as one of the main methodological tools in the Connectome project (Sporns, 2012).

Several interrelated questions arise in this context: what are the unique features of topological explanations? Are they different from mechanisms or just an abstract variety of them? If they are different, do they constitute an alternative explanatory strategy in certain domains or they are rather complementary to mechanistic approaches? Do topological explanations indicate a need to expand a conception of mechanistic explanations and in what 
ways? These are all still open philosophical question, and the present special issue aims at clearly formulating them, and proposing well-reasoned answers.

Despite the fact that this is the first collection of articles dedicated to the nature of topological explanation, a clear and coherent picture of topological explanation has begun to emerge. It is a picture about their features, varieties and their relations to mechanistic explanation. First, even though the mechanistic explanations are well understood and articulated in the literature, there are still ways to make the accounts a little bit clearer and even stronger, for example, in his contribution to this special issue Alex Rosenberg argues that the natural progression would be to generalize the definition of mechanistic explanation so that it can be applied across all sciences. On the other hand, Totte Harinen in his paper in this volume proposes to extend mechanistic interventionist account of counterfactuals so that it can include even the non-causal inter-level relations. When it comes to understanding the distinctness of topological explanation and their varieties, the papers in this special issue lay out a solid groundwork. Topological explanations abstract away from the details of the local elements in systems and instead focus on the very pattern of their interactions. Most importantly these features make the topological explanation uniquely applicable to certain phenomena, e.g. Ratkopf claims they are specifically applicable to non-decomposable systems, on the other hand Darrason argues that topological explanations are particularly well suited for explaining the genetic bases of certain diseases. Furthermore, the papers in this special issue show the wide-ranging plurality of ways in which mechanistic and topological explanations are combined in scientific practice in order to provide better, deeper and more comprehensive explanations of natural phenomena.

The key ideas and arguments are organized in a way that reflects the interconnectedness of these questions. We begin with two papers that propose distinct ways in which accounts of mechanistic explanation can be expanded (Rosenberg and Harinen), then two papers that discuss the distinct features of topological explanation (Rathkopf and Kostic) and one that discusses a more general variety of this kind of explanation, i.e. the structural explanations and their relation to mechanistic explanations (Felline), after that we have two papers that present ways in which mechanistic and topological explanations are used as complementary explanatory strategies (Huneman and Darrason).

In his contribution, Alex Rosenberg starts by pointing out a strategic methodological vagueness about ontic commitments in the programmatic literature on new mechanist philosophy. He claims that the central claims of this view, as formulated in MDC (2000) although not very interesting and really hard to disagree with, doesn't offer a clear way to find theses that conflict with this view. Rosenberg argues that proponents of the new mechanist philosophy, especially when it comes to their views about the nature of scientific explanation in cognitive and system's neuroscience, are so weak that it is difficult to conceive counterexamples to their claims. The key claim in Rosenberg's argument is that current accounts of the mechanist explanation commit the new mechanists to generalize the $3 \mathrm{M}$ (model-to-mechanism-mapping) requirement so that it can apply across all special sciences. He starts off with the influential Craver and Kaplan formulation of the $3 \mathrm{M}$ requirement, which is as follows:

"3M [for "model -to-mechanism-mapping requirement] In successful explanatory models in cognitive and systems neuroscience:

(a) the variables in the model correspond to components, activities, properties, and organizational features of the target mechanism that produces, maintains or underlies the phenomenon and 
(b) the (perhaps mathematical) dependencies posited among these variables in the model correspond to the (perhaps quantifiable) causal relations among the components of the target mechanism. [Craver and Kaplan, 2011, p. 611)."

In order to generalize it to all special sciences, Rosenberg argues, one only has to substitute "life sciences" for "cognitive and systems neuroscience" and put "model" in plural. The revised and generalized $3 \mathrm{M}$ requirement now looks like this:

"M. In successful explanatory models in the life sciences (including behavioral and social science)

(a) the variables in the model correspond to components, activities, properties, and organizational features of the target mechanism(s) that produces, maintains or underlies the phenomenon and

(b) the (perhaps mathematical) dependencies posited among these variables in the model correspond to the (perhaps quantifiable) causal relations among the components of the target mechanism(s).” (Rosenberg 2015, p 4).

Rosenberg asks. Once this generalization be made, are there good reasons to adopt the $\mathrm{M}$ requirement? Answers to these questions he thinks would make mechanisms much more interesting, in fact highly controversial.

He then proceeds to discuss various problems of such generalization. First one is the issue of causality and the autonomy of the higher-level explanations in biology. If one were to honour the $\mathrm{M}$ requirement, one would have to espouse eliminativism about dependencies at higher levels, denying that they are causal at all, and therefore not really explanatory in the mechanistic framework. In other words, in explanation that satisfies $M$, higher level dependencies in biology and other special sciences are destined to be eliminated by that very explanation.

On the other hand, Totte Harinen argues for an extended account of interventionism by first showing that the notion of mutual manipulability requires ideal interventions in models that contain causal as well as non-causal relations. He argues that typically non-causal dimension in mechanistic models is understood as a variety of supervenience or realization relation.

He then goes on to argue that the presence of these kinds of non-causal relations undermines the ideality of interventions on the model as a whole, and especially when it comes to top-down interventions. His way around this problem is to propose that the standard conditions for ideal interventions should be extended to capture non-causal relations in mechanistic models. His extended account of ideal interventions he calls the "causal inbetweeness". This idea is best understood as a three variable affair. Instead of traditionally conceived mutual manipulability between the higher level variable $\Psi$ and lower level variable $\Phi$, where interventions on $\Phi$ result in changes in $\Psi$, and that mutual manipulability requires ideal top down interventions from $\Psi$ to $\Phi$, Harinen's proposal is to invoke a third variable in this picture so that the interventions scheme involves higher level variables $\Psi$-in and $\Psi$-out, whose relation is some causal regularity, and which together are indicative of S's $\Psi$-ing. This picture of course still involves the lower level variable $\Phi$. The mutual manipulability on this proposal goes like this: one intervenes on the input variable $\Psi$-in and then observes if any changes occur in the value of lower-level variable $\Phi$. On the other hand, bottom-up intervention goes as before, one intervenes on the value of $\Phi$ and sees if some changes in the 
value of $\Psi$-out occur. The three variable understanding of mutual manipulability preserves the upward causation and supervenience of mechanisms upon their components. By extending Craver's mechanistic account of mutual manipulability to include a third variable, Harinen has shown that there is no special problem with non-causal relations in the metaphysics of mutual manipulability.

In order to be able to understand to a fuller extent the features and differences between mechanistic and topological explanations, and more generally the structural explanations, the next three papers discuss different aspects of topological explanation.

Charles Rathkopf in his contribution discusses two methodological theses. The first is that network science exploits the very properties that make a system complex in providing explanations. The second claim is that network representations are particularly useful in explaining non-decomposable systems. Taken together these two features make network explanation a truly novel explanatory strategy. His argument for the claim that topological explanations embrace complexity is the following. He provides a broad definition of a complex system as the one in which discrete elements display patterns of interaction that are non-trivial. Normally, when explaining complexity of such systems, the pattern of interactions among its elements plays a less important role in determining system's behaviour and because of that it is often suppressed or simplified with an all-purpose fixed parameter. For example, in ideal gases at equilibrium, molecular interactions are stochastically independent, and the exact pattern of their interactions can be ignored, which allows us to represent their interactions as a single distribution function. However, Rathkopf argues, such idealizations are not satisfactory because it is the particular pattern of interactions that plays ineliminable role in producing collective behaviour of a system. In such cases, to understand complexity of a system it is required to represent behaviour as a variable of the precise pattern of inter-element interaction. Such variable may be for example the exact mathematical value of the long-path connections and clustering coefficient in the Watts and Strogatz (1998) small-world graph model. This is the way in which network science actually embraces complexity rather than shying away from it.

The argument for his second claim, that topological explanations are particularly useful in explaining non-decomposable systems, starts from the very definition of a nondecomposable system. Rathkopf claims that a system is non-decomposable when the behaviour of any given component part depends on the behaviour of many other individual components. Given this definition it is easy to see that any network property is a relational property between a node or an edge and all other nodes or edges in a network. Charles uses a particularly good example of the topological property called "betweeness". Betweeness is a property of a single edge which is computed by examining every pairwise combination of nodes in the network and finding a shortest path length between each pair, which gives a data structure. The data structure obtained in this way is then searched to find the proportion of all paths that incorporate the value of the edge in question. That proportion is the property of betweeness of the edge. The betweeness of the network is then used to explain various phenomena, the most famous one is the explanation of urban traffic density. This example clearly shows how network properties are used in topological explanation to explain nondecomposable systems, such as urban traffic.

Taken together these two claims give a clear picture of why Rathkopf sees topological explanations as distinct, especially distinct explanatory strategy from mechanisms, it is because they: a) abstract away from the details of the component elements in systems and instead focus on the very pattern of their interactions, and b) because they are particularly well suited for explaining the non-decomposable systems, where mechanistic explanations normally break down.

On the other hand, Daniel Kostic, instead of focussing on the science of complexity, 
turns attention to topological approach in cognitive neuroscience and discusses the features of topological realization and its explanatory power.

In his contribution, Daniel Kostic develops an account of topological realization and explanation. He argues that the realization relation stands between a system and a topology, and he defines in the following way:

"(TR): The realization relation stands between a topology $T$ and a system $S$, such that the system $S$ realizes topology $T$ when the elements of $S$ are interconnected in ways that display the pattern of connectivity characteristic of $T$.

Elements in this definition can refer to some spatial objects (such as single neurons, species or brain regions) in spatially embedded networks, but as it is more often the case, it refers to some abstract representation of data, e.g. the voxels or brain states that are defined as magnitudes of neurophysiological activity or even points in a state space."

On his view the uniqueness of topological realization, compared to mechanistic and semantic accounts of realization, stems from the fact that the realization base is at the global level, regardless of the scales. He argues that topology represents patterns of connectivity in a system, and that any property of connectivity is defined in relation to the whole network, i.e. a hub is a vertex that is defined by the number of edges it maintains relative to other vertices in the network, or a path-length is defined as an average shortest path length connecting each vertex to all other vertices. This puts the realization base on the global level, regardless of the local nature of the elements that the network represents. This point becomes particularly evident when he compares the topological with the semantic realization. In semantic realization, the realization base is constituted by the microphysical descriptions that are always at the local level, for example the "watery stuff" is realized if it satisfies a a microphysical description $\mathrm{H} 2 \mathrm{O}$. This idea is embodied in the claim that all non-basic truths have to be a priori derivable from a set of microphysical descriptions. The derivation is only possible if the non-basic facts are shown to play the same causal or functional roles as the microphysical descriptions, thus defining the realization relation as a logical one where the properties are realized if they satisfy microphysical descriptions.

His second claim is that topological explanation is not based on the very fact that a system realizes certain topology. The topological explanation in his view is based on the ability to distinguish different realization bases. Different realization bases are distinguished by understanding the mathematical consequences of the topologies, and this in turn tells why that topology in particular is explanatory of the given explanandum. This is what makes the topological explanatory, and not merely descriptive.

Further distinction between mechanistic explanations and a more general account of structural explanation we find in the paper by Laura Felline.

In her contribution to this special issue Felline argues that that there are phenomena that are not mechanistically explained nor mechanistically explainable and yet they are explained by science. Her argument starts with the claim that although mechanistic explanation is the most successful and ubiquitous account of scientific explanation currently available, in the domain of fundamental physics we find examples of explanations of the phenomena that are not brute or primitive (i.e. they are not unexplainable facts of nature). Yet, these explanations are not really mechanistic. She calls these types of phenomena mechanistically brute, because they can't be explained mechanistically, but they are also not brute facts of nature. They are explained by using structural explanation. To understand this distinction it is important to note that at the lowest ontological level, the parts such as quantum objects do not produce higher level phenomena or behaviours by interacting among 
each other, nor does the their spatial location figure in way in the production of higher level properties or behaviours. In fact, quantum objects are not even describable as localized objects. Furthermore, quantum objects are almost always in entangled states, so it is difficult to individuate parts of the system with individual states, as the mechanistic explanation normally requires. Based on this, Felline argues, following Bokulich (2009), that structural explanation explains by showing how the explanandum counterfactually depends on mathematical features of the model instead of decomposing it to component parts. One such example is the geometrical explanation of relativistic effects in the special theory of relativity. Felline further points out a similarity between her account of structural explanation and Batterman's (2002) asymptotic explanation. The important difference between them, is not only in the use of technical terminology, e.g. what Batterman calls "physical" she calls "mechanical", but more importantly she claims that the structural explanation is not only an explanation of regularities, but also of individual phenomena. For all these reasons, she argues, the structural explanation is not only non-mechanistic, but it also rules-out the very possibility of mechanistic explanation in the domain of quantum phenomena. That being said, Felline sees some similarities between the mechanistic and structural explanation in the fact that they are both model based explanations and they both explain by providing accounts of counterfactual dependencies, even though the sources of these dependencies are different in each of the accounts. In the mechanistic explanation the explanandum phenomenon counterfactually depends on the ontic properties of the underlying mechanism, whereas in the case of the structural explanation the explanandum counterfactually depends on the variables that represent the mathematical features of the model.

Now, after the further details of topological and structural explanations are thoroughly presented, a discussion turns to issues of how they are combined with mechanistic explanations in actual scientific practice.

In his contribution Philippe Huneman develops an account of how mechanistic and topological explanations are combined within scientific practice, especially in biology and ecology. He starts off by defining topological explanations as a variety of structural explanations and arguing against a continuum view of explanations, which sees topological explanations as merely a very abstract kind of mechanisms. As he defines it, in a topological explanation, a feature, a trait, a property or an outcome of a system under focus is explained by the fact that it possesses specific topological properties. In this sense the explanandum is a mathematical consequence of certain topological properties. Based on this he claims that in topological explanation it is the topological facts that are explanatory, and not the diverse processes or activities that instantiate these topological properties. To further elaborate this point he makes a distinction between representational and explanatory role of mathematics in scientific models. Mechanistic models indeed use differential equations, transition probabilities and many other mathematical tools to characterize some processes or activities. But these are mathematical descriptions or representations of the causal or ontic details, that are actually explanatory. Yet in topological explanation it's not the processes or activities that are by themselves explanatory, i.e. accounting for the explanandum, but general topological properties they instantiate. Having distinguished the mechanistic and topological explanations, Huneman then discusses how they are combined in scientific practice. He argues that this is done in three ways, namely by mutual constraining, mutual conditioning and by combining into singular explanatory schemes.

In a general sense topologies may constrain the system dynamics, for example the lower connectivity in a network means that possible pathways between two points will be much longer which further means that the motions will be slower. Regarding how mechanistic and topological explanations condition each other, one can approach this issue by 
understanding the difference between the types of questions these two approaches are trying to answer. The third way in which they are combined is modelling which utilizes merging of networks of different natures to provide insights about the finer grained functioning of mechanisms. He concludes that the ways in which topological explanations develop provide powerful tools to make sense of plurality and richness of scientific practices.

Finally, in her contribution Marie Darrason makes three claims. First, she argues that topological explanation are indispensable in understanding disease in terms of robustness and functional redundancy. Second that topological and mechanistic explanations are different from one another in virtue of explaining the same phenomenon by appealing to different properties. And finally her third claim is that in medicine and medical genetics topological explanation provide a broader and more general view of the disease and the role of genetics in it, as opposed to mechanistic explanations that tend to focus on the specifics of particular diseases.

Her proposal is to abstract away from the roles of individual genes in individual diseases and look into the various ways "network medicine" is used in this area to get a more general explanation of the genetics of diseases that would also allow us to distinguish between monogenetic and polygenetic diseases.

The founding idea of this approach is that a perturbation of complex networks of inter and extracellular components in a tissue specific or organ specific systems allows us to define a disease as an interconnectivity property. This general approach is beneficial in numerous ways. Most notably for allowing us to identify pathological network of each disease as well as to distinguish which diseases share the same networks. In this way, Darrason convincingly shows numerous ways in which topological and mechanistic explanations are substantially different in their workings, but also why topological explanations are indispensable in understanding the genetic foundations of diseases. Topological explanations achieve this by providing a broader view of the role of genes in diseases and at the same time allowing us to find previously unknown interactions between genes, gene modules and diseases as well as to distinguish monogenic from polygenic diseases.

One major upshot of Darrason's contribution is that mechanistic and topological explanations should not be seen as competitors, but rather as complementary, especially in the case of network medicine. This is so, because even though the topological properties of a diseasome explain why the genes in the same disease module have a tendency to interact together, without specific mechanistic information that is represented in the diseasome (e.g. information about the protein-protein interactions, levels and scales of gene expressions) such explanation would not be complete.

This in turn is another example, from a different area of science, of Huneman's claim about devising a singular explanatory strategy by combining mechanistic and topological explanations.

The topological approach is still very young by all standards, and even within special sciences it still doesn't have a single methodological programme that is applicable across all areas of science. This fact reflects a tremendous richness and diversity of topological explanations that remains to be explored, both by the philosophers as well as by the scientists. That is why this special issue is important as a first systematic philosophical study of topological explanations and their relation to a well understood and widespread explanatory strategy, such as mechanistic one. 


\section{References:}

- Achard, S., Salvador, R., Whitcher, B., Suckling, J., \& Bullmore, E. D. (2006). A resilient, low-frequency, small-world human brain functional network with highly connected association cortical hubs. The Journal of neuroscience, 26(1), 63-72.

- Alexander-Bloch, A. F., Vértes, P. E., Stidd, R., Lalonde, F., Clasen, L., Rapoport, J., \& Gogtay, N. (2012). The anatomical distance of functional connections predicts brain network topology in health and schizophrenia. Cerebral cortex, bhr388.

- Blondel, V. D., Sontag, E. D., Vidyasagar, M., \& Willems, J. C. (Eds.). (2012). Open problems in mathematical systems and control theory. Springer Science \& Business Media.

- Barabási, A. L., Gulbahce, N., and Loscalzo, J. (2011). Network medicine: a network-based approach to human disease. Nature Reviews Genetics 12.1 (2011): 56-68.

- Barabasi, A.L. and Oltvai, Z. N. (2004). Network biology: understanding the cell's functional organization. Nature Reviews Genetics 5: 101-113.

- Barabasi, A. L. (2002). Linked: How everything is connected to everything else and what it means. Plume Editors.

- Barabási, A. L. and Albert, R. (1999). Emergence of scaling in random networks. Science 286.5439: 509-512.

- Batterman, R. W. and Rice, C. C. (2014). Minimal Model Explanations. Philosophy of Science, 81 (3), pp. 349-376.

- Batterman, R. W. (2009). On the explanatory role of mathematics in empirical science. The British Journal for the Philosophy of Science, axp018.

- Batterman, R. W. (2002). The devil in the details: Asymptotic reasoning in explanation, reduction, and emergence.

- Batterman, R. W. (2002). Asymptotics and the role of minimal models. The British Journal for the Philosophy of Science, 53(1), 21-38.

- Bassett, D. S., \& Siebenhühner, F. (2013). Multiscale network organization in the human brain. Multiscale Analysis and Nonlinear Dynamics: From Genes to the Brain, 179-204.

- Bassett, D. S., Greenfield, D. L., Meyer-Lindenberg, A., Weinberger, D. R., Moore, S. W., \& Bullmore, E. T. (2010). Efficient physical embedding of topologically complex information processing networks in brains and computer circuits. PLoS Comput Biol, 6(4), e1000748.

- Bassett, D. S., \& Bullmore, E. T. (2009). Human brain networks in health and disease. Current opinion in neurology, 22(4), 340.

- Bassett, D. S., Bullmore, E., Verchinski, B. A., Mattay, V. S., Weinberger, D. R., \& MeyerLindenberg, A. (2008). Hierarchical organization of human cortical networks in health and schizophrenia. The Journal of Neuroscience, 28(37), 9239-9248.

- Bechtel, W. and Richardson, R. C. (2010). Discovering complexity: Decomposition and localization as strategies in scientific research. Second Edition. Cambridge, MA: MIT Press/Bradford Books. 
- Bressler, S. L. (1995). Large-scale cortical networks and cognition. Brain Research Reviews, 20(3), 288-304.

- Bullmore, E., \& Sporns, O. (2009). Complex brain networks: graph theoretical analysis of structural and functional systems. Nature Reviews Neuroscience, 10(3), 186-198.

- Buck, J. (1988). "Synchronous rhythmic flashing of fireflies. II". Quarterly review of biology, 265-289.

- Bullmore, E. and Sporns, O. (2009). "Complex brain networks: graph theoretical analysis of structural and functional systems”. Nature Reviews Neuroscience 10, pp. 186-198.

- Bassett, Danielle S., and Edward T. Bullmore. (2009. Human brain networks in health and disease. Current opinion in neurology 22.4): 340.

- Bassett, D. S., \& Bullmore, E. D. (2006). Small-world brain networks. The neuroscientist, 12(6), 512-523.

- Chalmers, D. (2010). The Two-Dimensional Argument Against Materialism. In: McLaughlin B. P. and Walter S., eds., 2010. Oxford Handbook to the Philosophy of Mind. Oxford: Oxford University Press.

- Costa, L. D. F., \& Sporns, O. (2005). Hierarchical features of large-scale cortical connectivity. The European Physical Journal B-Condensed Matter and Complex Systems, 48(4), 567-573.

- Craver, C. F. (2015). The Explanatory Power of Network Models. Manuscript.

- Craver, C. F. 2013. "Functions and mechanisms: A perspectivalist view." Functions: Selection and mechanisms. Springer Netherlands, 133-158.

- Craver, C. F. (2007). Explaining the Brain: Mechanisms and the Mosaic Unity of Neuroscience. Oxford University Press, New York, USA.

- Cupal, J., Kopp, S., \& Stadler, P. F. (2000). RNA shape space topology. Artificial Life, 6(1), $3-23$.

- Darrason, M. (forthcoming). "Mechanistic and topological explanations in medicine: the case of medical genetics and network medicine." Synthese, 1-27.

- Dowell, J. (2008). A Priori Entailment and Conceptual Analysis: Making Room for Type-C Physicalism. Australasian Journal of Philosophy, 86: 93-111.

- Endicott, R. (2005): Multiple realizability. In The Encyclopedia of Philosophy. 2nd edn. New York: Macmillan.

- Fodor, J. A. (1974). Special sciences (or: the disunity of science as a working hypothesis). Synthese, 28(2), 97-115.

- Fortunato, S. (2010). Community detection in graphs. Physics Reports, 486 (3-5), pp. 75-174.

- Gillett, C. (2010). Moving beyond the subset model of realization: the problem of qualitative distinctness in the metaphysics of science. Synthese, 177(2), 165-192. 
- Gu, Shi, Fabio Pasqualetti, Matthew Cieslak, Qawi K. Telesford, B. Yu Alfred, Ari E. Kahn, John D. Medaglia et al. (2015). "Controllability of structural brain networks." Nature communications 6.

- He, Y., Chen, Z. J., \& Evans, A. C. (2007). Small-world anatomical networks in the human brain revealed by cortical thickness from MRI. Cerebral cortex, 17(10), 2407-2419.

- Honey, C. J., J. P. Thivierge, and Sporns, O. (2010). Can structure predict function in the human brain? Neuroimage 52 (3): 766-776.

- Honey, C. J., Kötter, R., Breakspear, M., \& Sporns, O. (2007). Network structure of cerebral cortex shapes functional connectivity on multiple time scales. Proceedings of the National Academy of Sciences, 104(24), 10240-10245.

- Hutchison, R. M., Womelsdorf, T., Allen, E. A., Bandettini, P. A., Calhoun, V. D., Corbetta, M., ... \& Chang, C. (2013). Dynamic functional connectivity: promise, issues, and interpretations. Neuroimage, 80, 360-378.

- Huneman, P. (2015). Diversifying the picture of explanations in biological sciences: ways of combining topology with mechanisms. Synthese, 1-32.

- Huneman P. (2010). Topological explanations and robustness in biological sciences. Synthese, 177, pp. 213-245.

- Jones, N. (2014). Bowtie structures, pathway diagrams, and topological explanation. Erkenntnis, 79(5), 1135-1155.

- Kaiser, M., \& Hilgetag, C. C. (2006). Nonoptimal component placement, but short processing paths, due to long-distance projections in neural systems. PLoS Comput Biol, 2(7), e95-e95.

- Kaplan, David M. (2011). Explanation and Description in Computational Neuroscience. Synthese 183:339-73.

- Klimm, F., Bassett, D. S., Carlson, J. M., \& Mucha, P. J. (2014). Resolving structural variability in network models and the brain. PLOS Comput Biol, 10(3), e1003491.

- Lange, M. (2013). What makes a scientific explanation distinctively mathematical? British Journal for the Philosophy of Science 64: 485-511.

- Leith, D. J., \& Leithead, W. E. (2000). Survey of gain-scheduling analysis and design. International journal of control, 73(11), 1001-1025.

- Levine, J. (2001). Purple Haze: The Puzzle of Conscious Experience. Cambridge, MA: MIT Press.

- Levy, A. and Bechtel, W. (2013). Abstraction and the Organization of Mechanisms. Philosophy of Science, 80 (2), pp. 241-261.

- Machamer, P., Darden, L. and Craver, C. F. (2000). Thinking about mechanisms. Philosophy of science, 67, pp. 1-25.

- McClelland, J. L. (1988). Connectionist models and psychological evidence. Journal of Memory and Language, 27(2), 107-123. 
- Meunier, D., Lambiotte, R., Fornito, A., Ersche, K. D., \& Bullmore, E. T. (2009). Hierarchical modularity in human brain functional networks. Frontiers in neuroinformatics, 3.

- Mirollo, R. E., \& Strogatz, S. H. (1990). Synchronization of pulse-coupled biological oscillators. SIAM Journal on Applied Mathematics, 50(6), 1645-1662.

- Muldoon, S. and Bassett, D. (2015). Network and Multilayer Network Approaches to Understanding Human Brain Dynamics, Manuscript.

- Newman, M. (2010). Networks: An Introduction. OUP Oxford.

- Pasqualetti, F., Zampieri, S., \& Bullo, F. (2014). Controllability metrics, limitations and algorithms for complex networks. IEEE Transactions on Control of Network Systems, 1(1), $40-52$.

- Polger, T. W. (2010). Mechanisms and explanatory realization relations. Synthese, 177(2), 193-212.

- Ponten, S. C., Daffertshofer, A., Hillebrand, A., \& Stam, C. J. (2010). The relationship between structural and functional connectivity: graph theoretical analysis of an EEG neural mass model. Neuroimage, 52(3), 985-994.

- Power, J. D., Cohen, A. L., Nelson, S. M., Wig, G. S., Barnes, K. A., Church, J. A., ... \& Petersen, S. E. (2011). Functional network organization of the human brain. Neuron, 72(4), 665-678.

- Rathkopf, C. (2015). Network representation and complex systems. Synthese DOI $10.1007 / \mathrm{s} 11229-015-0726-0$

- Silberstein, M., and Chemero, A. (2013). Constraints on localization and decomposition as explanatory strategies in the biological sciences. Philosophy of Science 80.5: 958-970.

- Seung, S. H. (2012). Connectome: How the Brain's Wiring Makes Us Who We Are. Houghton Mifflin Harcourt Trade, New York.

- Sporns, O., (2012). Discovering the Human Connectome. Cambridge, MA: MIT Press.

- Sporns, O., Tononi, G., \& Kötter, R. (2005). The human connectome: a structural description of the human brain. PLoS Comput Biol, 1(4), e42.

- Stadler, B. M., \& Stadler, P. F. (2004). The topology of evolutionary biology. In Modelling in Molecular Biology (pp. 267-286). Springer Berlin Heidelberg.

- Strogatz, S. (2003). Sync: The emerging science of spontaneous order. Hyperion, Chicago.

- Torre, V. (1976). A theory of synchronization of heart pace-maker cells. Journal of theoretical biology, 61(1), 55-71.

- van den Heuvel, M. P., \& Sporns, O. (2013). Network hubs in the human brain. Trends in cognitive sciences, 17(12), 683-696.

- Watts, D. J., and Strogatz, S. H. (1998). Collective dynamics of 'small-world' networks. Nature 393 (6684): 440-442. 
- Wilson, R. A., \& Craver, C. F. (2006). Realization: Metaphysical and scientific perspectives. Philosophy of psychology and cognitive science. North Holland, Amsterdam, 81-104.

- Woodward, J. (2013). Mechanistic explanation: its scope and limits. Aristotelian Society Supplementary Volume, 87. 1:39-65.

- Woodward, J. (2003). Making Things Happen: A Theory of Causal Explanation. New York: Oxford University Press. 